\title{
Reimagining Design Education in a new context
}

\author{
Keywords \\ Behaviours, Creative Process, Design, Education, Flow.
}

The impact Covid-19 had on people's lives over the last couple of years is still an evolving challenge and a field of ongoing research. The so-called 'new normal' or 'post-Covid world' seems to be more of an elusive, fluid, everchanging scenario rather than a stable reality as many initially expected or even hoped after the initial global pandemic outbreak. While unprecedented efforts from Governments and international organisms take place, experts in different disciplines -from epidemiologists to economists- are fully dedicated to researching, distilling insights and analysing results to not only assess and measure the actual 'live' impact of the -now turned endemic- pandemic yet also to forecast short and long-term implications. Yet, despite all this effort addressing the effect on the physical and mental wellbeing of individuals, communities and businesses, Educators are facing their specific challenge: teaching in an unknown and ever-changing paradigm. Adapting between intermittently teaching on-campus, remotely, and in hybrid models - mixing virtual and physical classrooms- Educators are finding themselves prototyping 'live' and testing in short sprints, attempting to adapt or even redesign pre-existing curriculums and methodologies. Is this working? What is the impact-tangible and intangible- in the learning outcomes and the students' experience? Is there even time for reflection, iteration and reassessment when the realities and context themselves are constantly shifting? Furthermore, there is an underlying challenge: how to keep students engaged 'remotely' when it is hard to understand the challenges they face in their 'home bubbles'? Reimagining Design Education in a new context is an article that explores how the new global dynamics impact specifically the Design education ecosystem and mainly focuses on how this affects the mindsets and emerging behaviours of Educators and young University Students (who are only becoming familiar with the Design world). Tracing parallels between Design education and industry-based practice, the exploratory journey in the manuscript takes into consideration foundational education, philosophical essays and incorporates different points of view -from Design Educators to concepts coming from Positive Psychology and Neuro-Linguistic Programming (NLP). Reimagining Design Education in a new context challenges the paradigms of core Design Education methodologies and aims to understand better the tensions between the current and potential future models and how this could impact the learning journey. Nevertheless, it also inspects how this relates to the Design Educators and Design Students' mindsets and behaviours beyond School, incorporating the challenges they face in this new non-conventional paradigm (including the students growing relationship with technology). As a piece of research, it does not offer final crystalised answers or solutions yet instead poses critical questions and offers an open dialogue with diverse points of view. 